\title{
Characterisation of DGAT1 and DGAT2 from Jatropha curcas and their functions in storage lipid biosynthesis
}

\author{
Ronghua $\mathrm{Xu}^{\mathrm{A}}$, Tianquan Yang ${ }^{\mathrm{A}, \mathrm{B}}$, Ruling Wang ${ }^{\mathrm{A}}$ and Aizhong Liu ${ }^{\mathrm{A}, \mathrm{C}, \mathrm{D}}$ \\ A Key Laboratory of Tropical Forest Ecology, Xishuangbanna Tropical Botanical Garden, Chinese Academy \\ of Sciences, 88 Xuefu Road, Kunming, 650 223, China. \\ ${ }^{B}$ The Graduate University of Chinese Academy of Sciences, Beijing 100049 , China. \\ CKunming Institute of Botany, Chinese Academy of Sciences, 132 Lanhei Road, Kunming, 650 204, China. \\ ${ }^{D}$ Corresponding author. Email: liuaizhong@mail.kib.ac.cn
}

\begin{abstract}
Diacylglycerol acyltransferases (DGATs) catalyse the final step of triacylglycerol (TAG) biosynthesis of the Kennedy pathway, and play a critical role during TAG accumulation in developing oleaginous seeds. In this study, the molecular cloning and characterisation of two DGAT genes, JcDGAT1 and JcDGAT2, from jatropha (Jatropha curcas L., a potential biodiesel plant) is presented. Using heterogonous overexpression techniques, both JcDGAT1 and JcDGAT2 were able to restore TAG biosynthesis in a yeast mutant H1246 strain, and enhance the quantity of TAG biosynthesis by 16.6 and $14.3 \%$, respectively, in strain INVSc1. In transgenic tobacco, overexpression of $J c D G A T 1$ and $J c D G A T 2$ resulted in an increase in seed oil content of, respectively, 32.8 and $31.8 \%$. Further, the functional divergence of $J C D G A T 1$ and $J_{C D G A T 2}$ in TAG biosynthesis was demonstrated by comparing the fatty acid compositions in both the transgenic yeast and tobacco systems. In particular, JCDGAT2 incorporated a 2.5-fold higher linoleic acid content into TAG than JCDGAT1 in transgenic yeast and exhibited a significant linoleic acid substrate preference in both yeast and tobacco. This study provides new insights in understanding the molecular mechanisms of DGAT genes underlying the biosynthesis of linoleic acids and TAG in plants.
\end{abstract}

Additional keywords: diacylglycerol acyltransferase, fatty acid composition, oil content, triacylglycerols.

Received 7 January 2013, accepted 1 October 2013, published online 19 November 2013

\section{Introduction}

Jatropha (Jatropha curcas L., Euphorbiaceae), which is commonly known as purging nut or physic nut, is an oilseedbearing perennial shrub native to Mexico and Central America. Owing to its biological properties such as drought hardiness, rapid growth, easy propagation, high oil content, short gestation period and wide adaptation to soil conditions, jatropha has created tremendous interest for the use of its seed oil (storage lipids) as a commercial source of biodiesel (Abdulla et al. 2011). However, worldwide introduction of jatropha as a biodiesel plant has had limited success because of unreliable oil yields and low economic returns. There is, therefore, an immediate need to enhance seed oil yields by genetic improvement of jatropha, which requires a comprehensive understanding and identification of the genes encoding the rate-limiting enzymes responsible for lipid biosynthesis in the developing seeds of in this species.

The biosynthesis of seed oils (in the form of triacylglycerols, TAGs) involves de novo fatty acid (FA) synthesis and TAG assembly. The process of de novo FA synthesis produces cytosolic acyl-CoAs in plasmids. The TAG assembly consumes acyl-CoAs using substrate glycerol 3-phosphate with diverse enzymes that sequentially transfer acyl-CoAs to sn-1, -2 and -3 positions in glycerol 3-phosphatein the endoplasmic reticulum (Ohlrogge and Browse 1995). There, acyl-CoA: diacylglycerol acyltransferase (DGAT; EC 2.3.1.20) transfers an acyl-CoA to the sn-3 position of sn-1, 2diacylglycerol (DAG) and plays an essential role in controlling TAG assembly (Shockey et al. 2006). At least two classes of genes, DGAT1 and DGAT2, encoding the DGAT enzymes have been identified in diverse plants (Zou et al. 1999; Giannoulia et al. 2000; Lardizabal et al. 2001; Nykiforuk et al. 2002; He et al. 2004; Yu etal. 2008; Li et al. 2010a). Results suggest that DGAT1 and DGAT2 are ubiquitous in plants. In Arabidopsis, AtDGAT1 controls TAG biosynthesis in developing seeds, whereas AtDGAT2 seems to have no obvious phenotype functionally (Shockey et al. 2006; Zhang et al. 2009). Similarly, the DGAT1s significantly regulate TAG accumulation in maize (Zheng et al. 2008), Tropaeolum majus L. (Xu et al. 2008) and soybean (Li et al. 2010b). Further, studies have shown that the DGAT2s play a critical role in the accumulation of unusual FAs (such as epoxy and hydroxy fatty acids) in castor bean (Kroon et al. 2006), tung tree (Shockey et al. 2006) and Vernonia (Li et al. 2010a). In addition, temporal and spatial expression patterns of DGAT1s and DGAT2s among organs or in different stages of seed development often display obvious 
differences (Li et al. 2010b; Banilas et al. 2011; Xu et al. 2011). However, little is known about the functional similarity or divergence of DGAT1 and DGAT2 during the pathway of storage lipid biosynthesis in planta.

In this study, we isolated and characterised DGAT1 and $D G A T 2$ from jatropha, and demonstrated their functions in storage lipid biosynthesis by heterologous expression in yeast (Saccharomyces cerevisiae) and tobacco systems. Results obtained from this study add new insights into our understanding of the molecular mechanisms of the oil content or fatty acid composition of TAG in seed oils of plants, and provide a step towards identifying the genes encoding the rate-limiting enzymes responsible for lipid biosynthesis in the developing seeds of jatropha.

\section{Materials and methods}

Plant material and yeast strain

Jatropha curcas L. was grown at the field station of Xishuangbanna Tropical Botanical Garden $\left(21^{\circ} 56^{\prime} \mathrm{N}\right.$, $101^{\circ} 15^{\prime} \mathrm{E}, 600 \mathrm{~m}$ above sea level), Chinese Academy of Sciences, Yunnan, SW China under natural conditions. Leaves, roots and seeds at different developmental stages were sampled as described in our previous study (Xu et al. 2011). Tobacco (Nicotiana tabacum L. cv. Honghua da jin yuan) was used as plant material for heterologous gene transformation. Both wild-type tobacco and its transgenic derivatives were grown in a greenhouse at $22^{\circ} \mathrm{C}$ with a 16 -h photoperiod $(16 \mathrm{~h}$ of light $120 \mu \mathrm{E} \mathrm{m}^{-2} \mathrm{~s}^{-1}$ ) and $8 \mathrm{~h}$ of darkness.

We used a yeast (Saccharomyces cerevisiae) quadruple mutant strain H1246 (Mato dgal::KanMX4 lro1::TRP1 are1:: HIS3 are 2::LEU2 ADE2 ura3) for complementary experiments (provided by Dr Sten Stymne, Swedish University of Agricultural Sciences, Uppsala). The H1246 strain lacks the DGA1 and LROI genes encoding yeast DGAT and PDAT enzymes responsible for the TAG synthesis, and ARE1 and ARE2 encoding ASAT (acyl-CoA: sterol acyltransferase) that are responsible for the synthesis of steryl esters (SE). Due to their absence, this mutant is devoid of the synthesis of TAG and SE, and does not form lipid droplets (LDs) (Sandager et al. 2002; Sorger et al. 2004). Therefore, this mutant is very useful for studying the enzymes of TAG synthesis, especially for identifying functional DGATs (Siloto et al. 2009). A wild-type yeast $S$. cerevisiae strain INVSc1 (Invitrogen, Guangzhou, China) was used as a control.
Cloning of DGAT1 and DGAT2 genes from Jatropha curcas

To isolate the DGAT genes from jatropha, total RNA was extracted from developing seeds as previously described (Xu et al. 2011). Complementary DNA was synthesised from $1 \mu \mathrm{g}$ of total RNA using a PrimeScript RT-PCR Kit (Takara Bio Co., Dalian, China). For the cloning of the DGAT1, specific primers, D1-HindIII and D1-Xho I (see Table 1) covering the open reading frame (ORF)), were designed based on an available jatropha DGAT sequence (DQ278448). The full length ORF of JcDGAT1 was amplified using high fidelity PCR TransStart FastPfu DNA Polymerase (Transgen, Beijing, China). The PCR product was cloned into a pEASY-Blunt Cloning Vector (Transgen) and the generated plasmid was designated pEasy-JcDGAT1.

For the cloning of DGAT2, the $5^{\prime}$ and $3^{\prime}$ RACE (rapid amplification of cDNA ends) methods were applied by using the Smart-RACE cDNA Amplification kit (Clontech Takara Biomedical Tech. Co., Beijing, China) according to the manufacturer's instructions. Gene specific primers, D2-51 and D2-31 (Table 1), were designed based on a partial sequence of jatropha DGAT (EU395774). The RACE-PCR products were analysed on a $1.2 \%(\mathrm{w} / \mathrm{v})$ agarose gel and the purified DNA was cloned into pEASY-T1 Cloning Vector (Transgen). Selected clones were purified and sequenced. After obtaining the fulllength cDNA of $J c D G A T 2$, the ORF was amplified with the primers D2-HindIII and D2-Xho I (see Table 1), and cloned into pEASY-Blunt Cloning Vector as described above. The generated plasmid was designated pEasy-JcDGAT2 and sequenced.

To investigate the structural characters of JcDGAT1 and $J C D G A T 2$ in the jatropha genome, DNA was extracted from jatropha leaves using the CTAB method and genomic PCR was performed using ORF primer pairs as described above. The reaction mixture $(50 \mu \mathrm{L})$ contained $100 \mathrm{ng}$ of genomic DNA template, $5 \mu \mathrm{L} 10 \times$ PCR buffer, $1 \mu \mathrm{M}$ of each primer, $0.25 \mathrm{mM}$ of dNTPs, and 2.5U of Transstart HiFi DNA polymerase (Transgen). The following PCR conditions were used: initial denaturation at $94^{\circ} \mathrm{C}$ for $3 \mathrm{~min}$, and 35 cycles of denaturation at $94^{\circ} \mathrm{C}$ for $30 \mathrm{~s}$, annealing at $55^{\circ} \mathrm{C}$ for $30 \mathrm{~s}$, and extension at $72^{\circ} \mathrm{C}$ for $5 \mathrm{~min}$. The PCR products were purified using silica column (Tiangen, Beijing, China) and sequenced. All sequencing was performed at Sangon Laboratories (Shanghai, China).

Table 1. Oligonucleotide primers used in this work

Restriction sites are underlined

\begin{tabular}{|c|c|}
\hline Primer & Sequence \\
\hline JcDGAT1-S-HindIII & 5'-GCGAAGCTTACCATGACGATTTTGGAGACCACT-3' \\
\hline JcDGAT1-A-XhoI & 5'-GCGCTCGAGTCATCTTAATTCAGCATTGCCTTTCCGA-3' \\
\hline$J_{c D G A T 2-51}$ & $5^{\prime}$-GACCACACCAAGGGGGAAAACTGAAT- $3^{\prime}$ \\
\hline$J_{C D G A T 2-31}$ & 5'-ATTCAGTTTTCCCCCTTGGTGTGGTC-3' \\
\hline JcDGAT2-S-HindIII & 5'-GCGAAGCTTACCATGGTAGGCGGAGATGGCAAT-3' \\
\hline JCDGAT2-A-XhoI & 5'-GCG $\overline{\overline{C T C G A G}}$ TCAAAGGATTTCAAGTTTAAGGT-3' \\
\hline NtActin-F & $5^{\prime}$-AAGGGATGCGAGGATGGA-3' \\
\hline NtActin-R & 5'-CAAGGAAATCACCGCTTTGG-3' \\
\hline
\end{tabular}




\section{Bioinformatical analysis}

Nucleotide sequences and amino acid sequences were primarily analysed using Vector NTI advanced software (ver. 11, Invitrogen Corporation, Carlsbad, CA, USA). Sequence homology searches in GenBank were conducted using BLAST (http://blast.ncbi.nlm.nih.gov/Blast.cgi, accessed 4 October 2011). AlignX was used to align DGAT amino acid sequences with the default settings, and further refined by visual inspection. Phylogenetic analysis was conducted using the neighbourjoining criteria in MEGA (ver. 5.0; Tamura et al. 2011). Branch support of the phylogenetic tree was estimated on the basis of 1000 bootstrap replicates of the data. Transmembrane domains of proteins were predicted using the TMHMM Server ver. 2.0 (http://www.cbs.d.tu.dk/services/TMHMM/, accessed 11 September 2011) and subcellular localisation of the deduced polypeptide was estimated using PSORT (http://psort. ims.u-tokyo.ac.jp/form.html, accessed 10 September 2012). The genomic structure of both $J_{C D G A T 1}$ and JcDGAT2 were identified using the Spidey server (http://www.ncbi.nlm.nih. gov/spidey/, accessed 5 September 2011) and schematised using the Gene Structure Display Server (http://gsds.cbi.pku. edu.cn/index.php, accessed 5 September 2011).

Heterologous expression and fatty acid substrate feeding in yeast

For expression of JCDGAT1 and JcDGAT2 in yeast, pEasyJcDGAT1 and pEasy-JcDGAT2 were digested with HindIII and Xho I, and the ORFs of JcDGAT1 and JcDGAT2 were respectively cloned into the p426-GAL vector with a GAL1 promoter (Mumberg et al. 1995), generating vectors p426JcDGAT1 and p426-JcDGAT2. The cloned sequences were verified by sequencing. Subsequently, p426-JcDGAT1 and

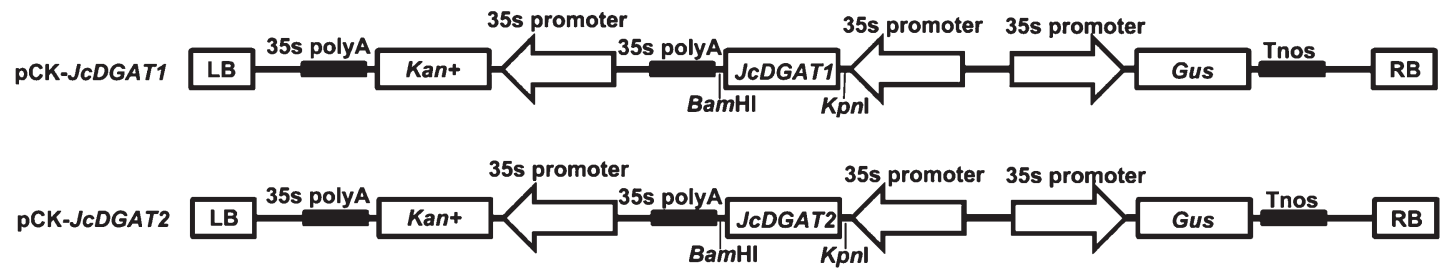

Fig. 1. Schematic structures of the T-DNA regions of pCK-DGAT1 and pCK-DGAT2 vectors for overexpression of $J c D G A T 1$ and JcDGAT2 in tobacco.

(a)

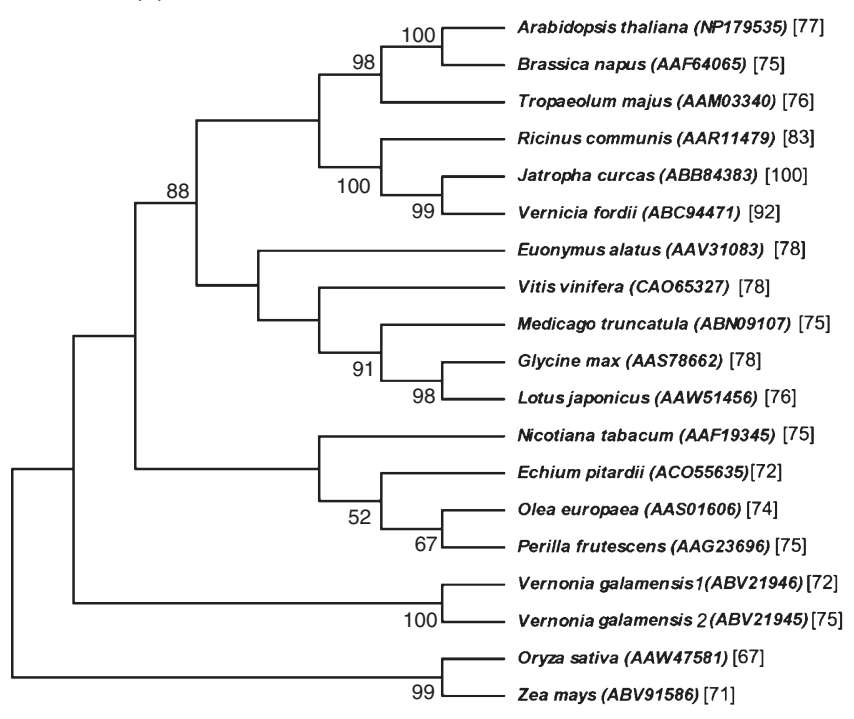

(b)

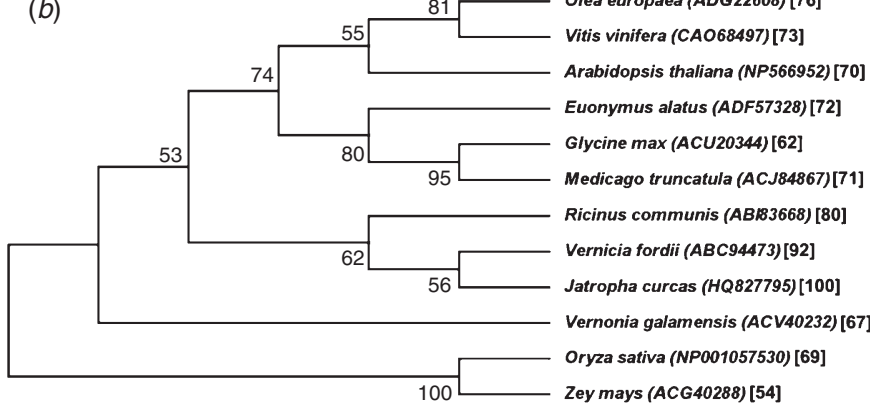

(c)

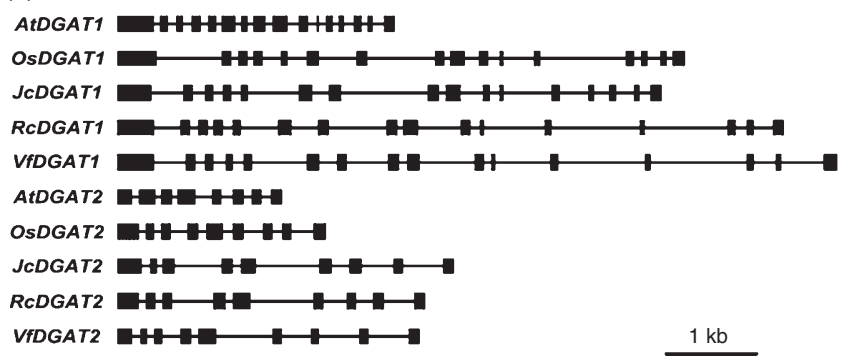

Fig. 2. Phylogenetic relationships and their genomic structures of plant $D G A T 1$ and $D G A T 2$ genes. The trees were reconstructed with the neighbour-joining criteria. GenBank accession numbers are given in parentheses. Numbers above branch nodes are bootstrap values from 1000 replicates. The 0.1 scale represent $10 \%$ divergence, calculated as estimate numbers of replacement. Only values $\geq 50 \%$ are present. Numbers within brackets correspond to $\%$ homologies between jatropha and other species within each group. (a) Phylogenetic tree of DGAT1; (b) phylogenetic tree of DGAT2; (c) genomic structure of plant DGAT1 (AtDGAT1, NM_127503, OsDGAT1, NC_008398, JcDGAT1, JQ3198121, RcDGAT1, NW_002994288, VfDGAT1, DQ356679) and DGAT2 (AtDGAT2, NC_003074, OsDGAT2, NC_008399, JcDGAT2, JQ3198123, RcDGAT2, NW_002994600,_VfDGAT2, DQ356681) genes. The filled boxes indicate exons and the lines indicate introns. 
p426-JcDGAT2 were transformed into H1246 and INVSc1, respectively, using the S. EasyComp Transformation Kit (Invitrogen). Yeast strains H1246 and INVSc1 harboured the p426-GAL empty vector and were used as negative and positive controls. Transformants were selected and expression of the recombinant genes in yeast was induced as previously described (Xu et al. 2008). After induction, cultures were grown at $30^{\circ} \mathrm{C}, 250 \mathrm{rpm}$.

To test whether the DGATs encoded by JcDGAT1 and $J_{C D G A T 2}$ exhibit substrate preference in yeast cells, the transgenic yeast cells were fed on linoleic acid (18C:2) by exogenously adding linoleic acid into media as acyl substrate of DGAT action. Specifically, the transgenic yeast cell culture was induced at an OD600 of 0.4 by supplementing galactose to $2 \%(\mathrm{w} / \mathrm{v})$. Linoleic acid was subsequently added into the media to a final concentration of $200 \mathrm{mmol} \mathrm{L}^{-1}$ with $1 \%(\mathrm{w} / \mathrm{v})$ Tergitol NP-40 (Sigma-Aldrich, Guangzhou, China) for making linoleic acid dissolve completely. After a $24 \mathrm{~h}$ incubation period, yeast cells $(20 \mathrm{~mL})$ were collected by centrifugation, washed with $0.1 \mathrm{M} \mathrm{NaHCO}$ and stored at $-20^{\circ} \mathrm{C}$ for further lipid analysis.

\section{Yeast lipid analysis}

Total lipid (TL) content of yeast cells was spectrophotometrically assayed using sulfophospho-vanillin methods (Izard and Limberger 2003; Wang et al. 2009). A total of $50 \mu \mathrm{L}$ of yeast cells at stationary phase were used for vanillin assay, and $1950 \mu \mathrm{L}$ of yeast cells were used for dry weight quantisation. Standard curves for converting absorbency readings into quantities of lipids were obtained from two replicate series of experiments, in which the absorbency of known concentrations of each reserve was measured.

To analyse the lipid composition of the yeast, TLs were extracted using a hexane/isopropanol (3:2, v/v) method, as previously described (Xu et al. 2011), from $20 \mathrm{~mL}$ of different yeast strain cells at the stationary growth phase. The TL was then dissolved in $500 \mu \mathrm{L}$ of hexane and the neutral lipids (NLs) were separated by one-dimensional TLC using silica gel 60 TLC plates (Haiyang, Qingdao, China). Plates were activated in an oven at $120^{\circ} \mathrm{C}$ for $1 \mathrm{~h}$ before use. The sample spots were developed with hexane/diethyl ether/acetic acid ( $80: 20: 1$, v/ $\mathrm{v} / \mathrm{v}$ ) and NL classes were visually determined using iodine staining and identified using aco-chromatograph with authentic standards. Fatty acid methyl esters (FAMEs) were prepared from the TAG spot and analysed as previously described (Xu et al. 2011).

\section{Nile red staining and microscopy}

Intracellular lipid bodies as an indicator for TAG formation were visualised by fluorescent microscopy using Nile red (Invitrogen) staining as described previously (Wagner et al. 2010). Aliquots of yeast cells $(500 \mu \mathrm{L})$ grown to stationary phase were harvested, washed twice with $1 \times \mathrm{PBS}$, dissolved in $500 \mu \mathrm{L}$ of $1 \times$ PBS, stained with $10 \mu \mathrm{L}$ Nile red dye $\left(2 \mathrm{mg} \mathrm{mL}^{-1}\right.$ in DMSO), and then incubated in a dark room for $15 \mathrm{~min}$ at room temperature. The stained cells were washed twice with $1 \times$ PBS and resuspended in $200 \mu \mathrm{L}$ distilled water, and analysed using fluorescence microscopy (Olympus BH2-UMA, Optical Co., LTD, Tokyo, Japan).

\section{Plant transformation}

The ORFs of JcDGAT1 and JcDGAT2 were subcloned into the KpnI-BamHI sites of a pCambia 2301-101 binary vector with the $35 \mathrm{~S}$ cauliflower mosaic virus promoter, and named pCKJCDGAT1 and pCK-JcDGAT2 (see Fig. 1). These vectors also carry the nptII gene coding for neomycine phosphotransferase conferring kanamycin resistance and the $\beta$-glucuronidase (GUS) gene. The recombinant plasmids (35S/JcDGAT1/nos, $35 \mathrm{~S} / J c D G A T 2 /$ nos) were transformed into wild-type tobacco by Agrobacterium tumefaciens (strain GV3101) mediated transformation using the modified leaf-disk method (Horsch et al. 1985). Transgenic plants $\left(\mathrm{T}_{0}\right)$ were selected and analysed as previously described (Worrall 1998). DNA and RNA were extracted from $100 \mathrm{mg}$ of tobacco leaf material. Successful transformation and the presence of the targeted genes were confirmed using GUS histochemistry analysis and PCR amplification of genomic DNA with primers specific to JcDGAT1 and JcDGAT2.

\section{GUS histochemistry}

Seedlings of different transgenic lines and the wild-type tobacco were incubated in a solution of $100 \mathrm{mM}$ potassium phosphate, $\mathrm{pH}$ 7.0, $10 \mathrm{mM}$ EDTA, 0.1\% Triton X-100, $1 \mathrm{mM}$ 5-broma-4chloro-3-indolyl- $\beta$-D-glucuronide for $24 \mathrm{~h}$ at $37^{\circ} \mathrm{C}$. The tissues were decolourised with $70 \%$ ethanol and photographed with a digital camera (DMC-FZ100GK, Panasonic, Japan).

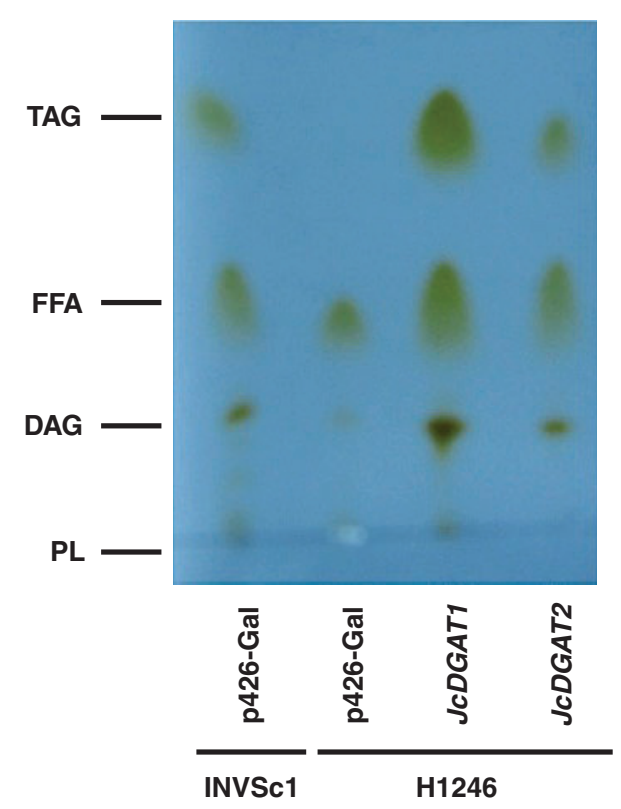

Fig. 3. TLC separation of neutral lipids collected from different yeast strains. The transgenic H1246 strains with the JcDGAT1 and JcDGAT2 restore triacylglycerol (TAG) synthesis, compared with controls. The amount of diacylglycerol (DAG), free fatty acid (FFA), phosphatidic lipid $(\mathrm{PL})$ and TAG were visualised by iodine staining. Presented is a representative result of three independent experiments. 
(a)

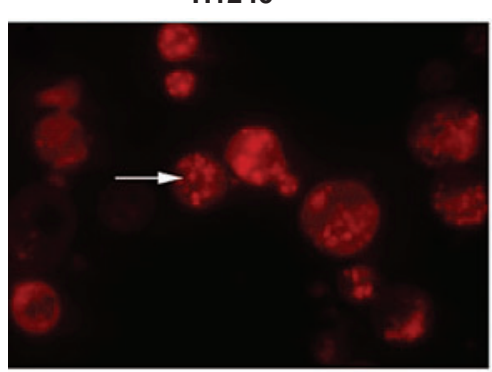

(b)

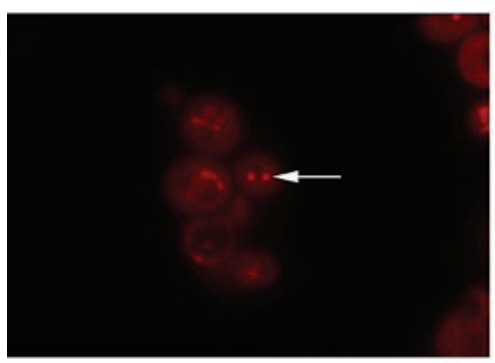

(c)

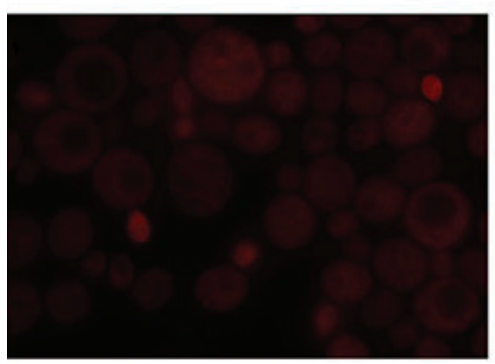

(d)

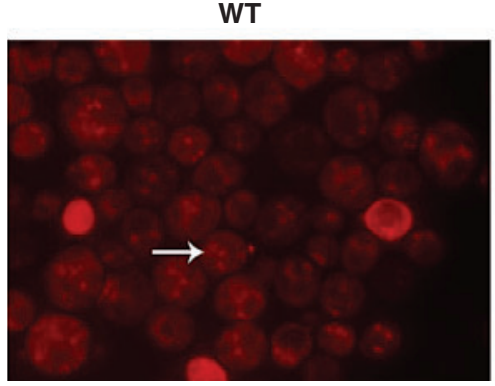

H1246
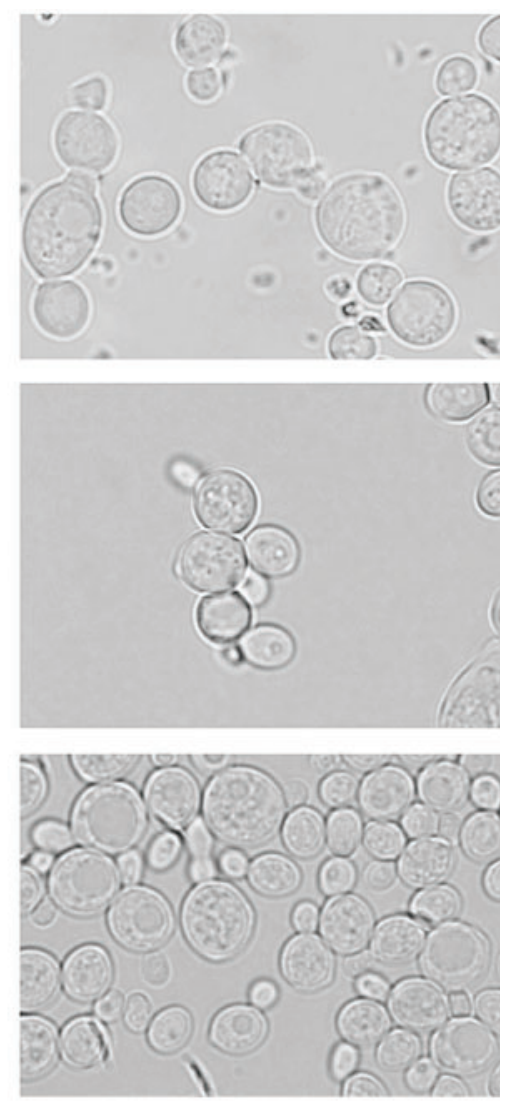

WT

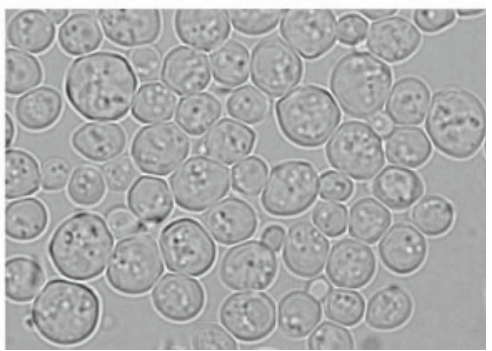

Fig. 4. Formation of lipid bodies is restored upon expression of $J_{C D G A T 1}$ and JCDGAT2. Triacylglycerol (TAG) accumulation in lipid bodies was visualised in yeast cells using the fluorescent dye Nile Red as described in Materials and methods. The TAG-deficient quadruple mutant strain H1246 was expressing either $J_{c} D G A T 1(a)$, or $J_{c} D G A T 2(b)$. As negative control the mutant strain $\mathrm{H} 1246$ harbouring the empty plasmid was analysed $(c)$. As positive control the wild-type strain INVSc1 was analysed $(d)$. The left panel images showed the yeast cells under the fluorescence microscopes and the right panel images showed the yeast cells under optical microscope. Presented is a representative result of three independent experiments.

Seed weight determination and lipid analysis

To determine average seed weight, four replicates of 50 tobacco seeds randomly counted from each line were weighed. These seeds were dried in open tubes in desiccators for $24 \mathrm{~h}$ before weighing and counting (Maisonneuve et al. 2010). For lipid analysis of transgenic tobacco, four aliquots of 50 tobacco seeds from each line were homogenised three times $(15000 \mathrm{rpm}, 30 \mathrm{~s}$ each) in $800 \mu \mathrm{L}$ hexane/isopropanol $(3: 2$, v/v, $0.01 \%$ BHT) mixture with a superfine homogenizer (Fluko Equipment Shanghai Co., Ltd, Shanghai, China). After dispersion, the homogenate was subjected to direct transmethylation as described by Maisonneuve et al. (2010). This method involves adding $2 \mathrm{~mL}$ of methanol containing $2.5 \% \mathrm{H}_{2} \mathrm{SO}_{4}(\mathrm{v} / \mathrm{v})$ to each sample and heating at $90^{\circ} \mathrm{C}$ for $90 \mathrm{~min}$. In addition, we used a heptadecanoic acid internal standard at a final concentration of $5 \mathrm{ng} \mu \mathrm{L}^{-1}$ for quantification. 
After cooling, $500 \mu \mathrm{L}$ of hexane and $2.5 \mathrm{~mL}$ of $500 \mathrm{mM} \mathrm{Na}_{2} \mathrm{SO} 4$ were added. FAMEs were extracted into the hexane phase by vigorous shaking followed by centrifugation at $1500 \mathrm{~g}$ for $5 \mathrm{~min}$. GC analysis of FAMEs were carried out as previously described (Xu et al. 2011). Individual methyl esters were identified by comparison with standards (Sigma-Aldrich). Fatty acid mehyl esters and total lipids were calculated by comparing with the heptadecanoic acid methyl ester standard.

\section{Results}

Cloning, gene structure and phylogenetic analysis of JCDGAT1 and JcDGAT2

The full length ORFs of the JcDGAT1 and JcDGAT2 (HQ827795) contain 1563 and 1373 nucleotides, encoding 521 and 352 amino acids respectively. The amino acid sequences of JcDGAT1 and JcDGAT2 are 67 and 56\% similar to the corresponding orthologues from Arabidopsis (At2 g19450 and At3 g51520, see Stone et al. 2006; Liu et al. 2011).

Genomic structure analysis showed that JCDGAT1 (JQ319812) contains 16 exons and 15 introns, while JcDGAT2 (JQ319813) contains nine exons and eight introns. Further, we compared the genomic structures of JcDGAT1 and JcDGAT2 with DGAT1s and DGAT2s identified from other plants, and found that the number of introns/exons is same or similar (Fig. 2c), suggesting that splicing patterns of DGAT1 and $D G A T 2$ are conserved in plants. Predictions of transmembrane domain (TMD) and subcellular localisation showed that both JcDGAT1 and JcDGAT2 were located in the endoplasmic reticulum. A blast search against the jatropha genomes released (http://www.kazusa.or.jp/jatropha/, accessed 3 March 2012) revealed that both JcDGAT1 and JcDGAT2 were represented by a single copy in jatropha genomes. Phylogenetic analysis showed both JcDGAT1 (see Fig. 2a) and JcDGAT2 (see Fig. 2b) are closely related to their allies cloned from other members (such as castor bean and tung tree) in Euphorbiaceae, reflecting their evolutionary homology.

\section{Functional analyses of JcDGAT1 or JcDGAT2 in yeast}

After $J_{C D G A T 1}$ and $J_{C D G A 2}$ were heterologously expressed in the yeast quadruple mutant strain H1246, The TLC fraction analysis showed that both $J c D G A T 1$ and $J c D G A T 2$ transgenic H1246 strains presented TAG spots (see Fig. 3), indicating that the DGAT enzymes encoded by $J c D G A T 1$ and $J_{C D G A T 2}$ were able to restore TAG biosynthesis in yeast. As shown in Fig. 4, the subcellular oil bodies were visualised in both JCDGAT1 and $J C D G A T 2$ transgenic cells under a fluorescent microscope. Moreover, the number of oil bodies visualised within a single $J C D G A T 1$ transgenic cell is more than that within a single JcDGAT2 transgenic cell, implying that the JcDGAT1 transgenic cells produced more TAG than the JcDGAT2 transgenic cells.

The oil content of cell dry weight (including TLs and NLs) in the transgenic H1246 and wild-type INVSc1 lines was shown in Fig. 5. Within the H1246 strain, the oil content of TLs and NLs in both $J_{C D G A T 1}(13.2$ and 9.1\%) and JCDGAT2 (10.6 and 7.1\%) transgenic $\mathrm{H} 1246$ cells were significantly higher $(P<0.01)$ than the control. Within the INVSc1 strain, the oil content of TLs and NLs in both $J c D G A T 1$ and $J c D G A T 2$ overexpressed cells were significantly higher $(P<0.05)$ than the control. In particular, within both H1246 and INVSc1 transgenic strains, JcDGAT1 produced significantly higher oil content of both TLs and NLs than JcDGAT2. These results clearly indicate that DGATs

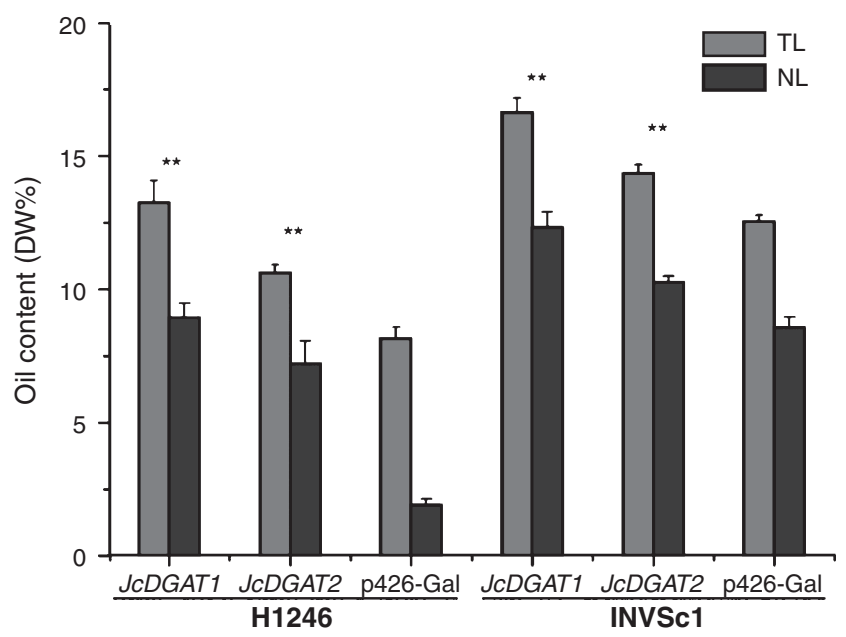

Fig. 5. Oil content analyses of strains H1246 and INVSc1 transformed with $J C D G A T 1, J C D G A T 2$ and the empty vector p426-gal respectively. The extraction of total lipids (TLs) from different yeast strains, the isolation of neutral lipids (NLs), and their estimation were described in 'Materials and methods'. Presented here is a representative result of three independent experiments with standard deviation as indicated by the error bars: $P$ values are: $*, P<0.05 ; * *, P<0.01$.

Table 2. Comparison of fatty acid composition of TAGs accumulated from transgenic yeast H1246 strains of JcDGAT1 and JcDGAT2, and their culture fed with linoleic acid (JcDGAT1-C18:2 and JcDGAT2-C18:2 respectively)

Results are representative of three independent experiments. FAs, fatty acids

\begin{tabular}{lcccr}
\hline & JcDGAT1 & JcDGAT1-C18:2 & JcDGAT2 & JcDGAT2-C18:2 \\
\hline C16:0 & $10.9 \% \pm 0.9 \%$ & $11.9 \% \pm 1.2 \%$ & $6.4 \% \pm 1.0 \%$ & $7.5 \% \pm 0.9 \%$ \\
C16:1 & $27.7 \% \pm 1.2 \%$ & $25.2 \% \pm 2.4 \%$ & $29.8 \% \pm 5.8 \%$ & $24.7 \% \pm 0.7 \%$ \\
C18:0 & $9.9 \% \pm 0.6 \%$ & $10.1 \% \pm 0.7 \%$ & $7.4 \% \pm 0.2 \%$ & $7.2 \% \pm 0.5 \%$ \\
C18: 1 & $51.5 \% \pm 1.8 \%$ & $42.2 \% \pm 0.9 \%$ & $56.4 \% \pm 4.7 \%$ & $35.7 \% \pm 1.5 \%$ \\
C18:2 & - & $10.6 \% \pm 0.8 \%$ & - & $25 \% \pm 2.6 \%$ \\
Total saturated FAs & $20.8 \%$ & $22 \%$ & $13.8 \%$ & $14.7 \%$ \\
Total unsaturated FAs & $79.2 \%$ & $78 \%$ & $86.2 \%$ & $85.4 \%$ \\
\hline
\end{tabular}


encoded by $J_{C D G A T 1}$ and $J_{C D G A T 2}$ were able to restore TAG biosynthesis, and that $J_{C D G A T 1}$ produced higher oil content within transgenic yeast cells than $J c D G A T 2$.

As Table 2 shows, comparisons of fatty acid compositions of TAG accumulated within transgenic H1246 cells revealed that the $J_{C} D G A T 1$ transgenic cells produced significantly higher $(P<0.01)$ saturated fatty acids $(20.8 \%)$, including 16C:0 and $18 \mathrm{C}: 0$, than the JcDGAT2 transgenic cells (13.8\%). Since yeast TAG lacks linoleic acid (Sandager et al. 2002), the substrate preference of DGATs encoded by JcDGATland JcDGAT2 could be determined by feeding external linoleic acid. Linoleic acid incorporated within the JcDGAT2 transgenic H1246 cells (25\%) was 2.5-fold greater than in $J C D G A T 1$ transgenic strains
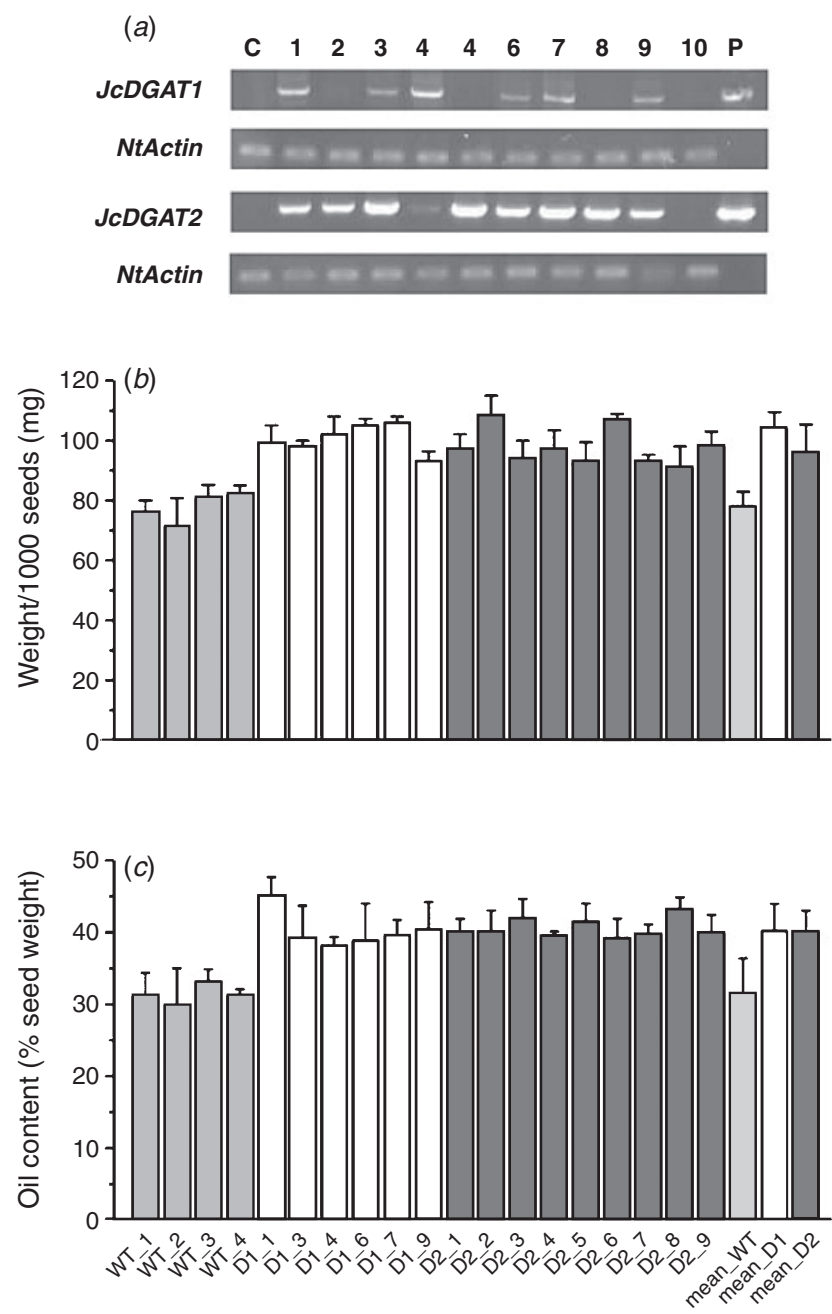

Fig. 6. RT-PCR analysis and comparison of seed weight and oil content among transgenic tobacco lines and the controls. (a) RT-PCR analyses of 10 $J_{C D G A T 1}$ or $J_{C D} D A T 2$ transformants in transgenic lines and controls ( $\mathrm{C}$ and $\mathrm{P}$ denote the negative and positive control, respectively, the actin gene of tobacco was used as a housekeeping control). Based on the presence of the targeted genes in RT-PCR analyses (a), the six and nine successful transformants of JCDGAT1 and JCDGAT2 were sorted out respectively. The seed weight $(b)$ and oil content $(c)$ of JcDGATland JcDGAT2 transformants and four wild types were compared with their mean, mean_D1, mean_D2, mean_WT respectively.
(10.6\%), suggesting the JcDGAT2 has a linoleic acid substrate preference than the JcDGAT1. The lower oleic acid content in $J c D G A T 2$ transgenic cells (35.7\%) than in JcDGAT1 transgenic cells $(42.2 \%)$ might be due to substrate competition resulting in the reduction of oleic acid during TAG biosynthesis.

\section{Functional analyses of JCDGAT1 or JcDGAT2 in planta}

After the transformants were determined by GUS analysis and RT-PCR amplification, six and nine independent transgenic lines of $J_{C D G A T 1}$ and $J_{C D G A T 2}$ were identified and cultivated in the greenhouse. Five non-transformed wild-type plants were cultivated as controls. Their seeds $\left(\mathrm{T}_{0}\right)$ were harvested to analyse. Seed oil content and weight were compared between different transgenic plants and the control (see Fig. 6). Both $J_{C D G A T 1}$ and $J_{C D G A T 2}$ transgenic plants showed that $\sim 32 \%$ increased oil content, and seed weight increased by 33 and $23 \%$ respectively. However, there was no significant difference between seed oil content and weight of JcDGAT1 and JCDGAT2 transgenic plants. The total saturated/unsaturated FA content did not make a significant difference among the $J_{c} D G A T 1, J_{c} D G A T 2$ transformants and the controls (Table 3). But, the content of the $18 \mathrm{C}: 2 \mathrm{FA}$ in the $J_{c D G A T 2}$ transformants $(73.85 \%)$ increased significantly compared with the $J c D G A T 1$ transformants (66.60\%) and the control $(67.12 \%)$, correspondingly, the 18C:1 FA $(11.08 \%)$ significantly decreased. This suggests that $J_{c} D G A T 1$ and $J c D G A T 2$ make different contributions to the accumulation of $18 \mathrm{C}: 2 \mathrm{FA}$ in tobacco seeds.

\section{Discussion}

DGAT is the only enzyme in the Kennedy pathway that is thought to be exclusively committed to TAG biosynthesis, and thus it is considered a key enzyme in this reaction (Lung and Weselake 2006). In addition to DGAT1 and DGAT2 (which have been extensively identified in plants), the third class DGAT3 gene was identified from Arabidopsis (Hernández et al. 2012) and peanut (Saha et al. 2006), but little is known about the DGAT3 gene in other plants. Studies have shown that the DGAT1 and DGAT2 represent different gene families with its own functional motifs in gene structure. Why the DGAT1 and DGAT2 present similar

Table 3. Comparison of fatty acid composition of seed oils accumulated from the transformants of $J c D G A T 1$ and JcDGAT2 in tobacco Presented here is the mean of four individual wild types (WT), six individual $J_{C D G A T 1}$ transformants and nine individual JCDGAT2 transfomrants. FAs, fatty acids. $P$-values are: ${ }^{*}, P<0.05$; $^{*}, P<0.01$

\begin{tabular}{lccc}
\hline & WT $(\%)$ & JcDGAT1 (\%) & JcDGAT2 (\%) \\
\hline C14:0 & $0.3 \pm 0.1$ & 0.3 & $0.5 \pm 0.2$ \\
C16:0 & $10.4 \pm 1.2$ & $10.1 \pm 1.7$ & $9.6 \pm 0.7$ \\
C18:0 & $5.4 \pm 1.1$ & $3.9 \pm 1.2$ & $3.6 \pm 0.8$ \\
C16:1 & $0.2 \pm 0.1$ & $0.2 \pm 0.1$ & 0.2 \\
C18:1 & $15.1 \pm 1.4$ & $16.8 \pm 1.8$ & $11.1 \pm 1.3^{*}$ \\
C18:2 & $67.1 \pm 1.3$ & $66.6 \pm 1.2$ & $73.9 \pm 1.5^{* *}$ \\
Total saturated FAs & 16.1 & 15.3 & 13.7 \\
Total unsaturated FAs & 82.4 & 83.6 & 85.1 \\
others & $1.5 \pm 0.5$ & $1.1 \pm 0.3$ & $1.2 \pm 1.2$ \\
\hline
\end{tabular}


function in biosynthesis of TAG in plants is, however, not well understood (Li et al. 2010b; Turchetto-Zolet et al. 2011).

Our results show that both $J_{c} D G A T 1$ and $J_{C D G A T 2}$ were able to restore TAG synthesis in the yeast cell and tobacco, reflecting the functional similarity of JcDGAT1 and JcDGAT2 in TAG biosynthesis. However, the oil contents of JcDGAT1 and JcDGAT2 transgenic yeast cells made significant differences, implying JcDGAT1 has a greater contribution to regulation of TAG quantity during the pathway of TAG biosynthesis. However, the seed oil content and weight of JCDGAT1 and JCDGAT2 transformants in tobacco did not present a significant difference in our current study. This may be because we omitted screening the homozygous transgenic seeds for each transgenic line, meaning that results obtained were the average values from different transgenic genotypes such as homozygous, hemizygous, or non-transgenic seeds. The non-transgenic and hemizygous seeds could decrease the difference between the oil content of JCDGAT1 or $J_{C D G A T 2}$ transgenic lines. The oil content and weight of homozygous transgentic seeds might be higher than our current average values.

In addition, we demonstrated that JcDGAT2 had an obvious substrate preference to linoleic acid in both yeast and tobacco systems, compared with JcDGAT1. To our knowledge, this is the first report on the substrate preference of DGAT2 to linoleic acid in plants. Previous studies also showed that RcDGAT2, $V g D G A T 2$ and VfDGAT2 played a major role in TAG assembly of hydroxy or epoxy fatty acids in developing seeds (Shockey et al. 2006; Burgal et al. 2008; Li et al. 2010b). It seems that DGAT2s have a greater contribution to accumulation of specific fatty acid compositions of TAG in planta than $D G A T 1$ s. The major function of DGAT2s may be for regulating or controlling the fatty acid composition of TAG by selectively incorporating different acyls in plants. However, it is uncertain whether the substrate preference of DGAT2 to linoleic acid is species dependent or ubiquitous in plants. If it is jatropha-species dependent, JcDGAT2 could be used as a gene resource for altering fatty acid composition of TAG by genetic engineering. Further investigation on overexpression or silence of $J_{C D G A T 1}$ and $J_{C D G A T 2}$ is necessary to determine their functions in regulating TAG biosynthesis during jatropha seed development.

\section{Conclusions}

Our data characterised the structure and function of $J_{C D G A T 1}$ and $J c D G A T 2$, and demonstrated their functions in regulating the quantity of TAG and fatty acid composition of TAG during TAG biosynthesis in both yeast and tobacco systems. These results provide new insights into understanding the molecular mechanisms underlying the extensive difference of oil content or fatty acid composition of TAG in seed oils.

\section{Acknowledgements}

We thank Dr Sten Stymne (Swedish University of Agricultural Sciences, Uppsala) for kindly providing the H1246strain. This research was jointly supported by NSFC (Grant No.30871548 and 31300569), and the 'Hundred Talents Program of the Chinese Academy of Sciences' to AL.

\section{References}

Abdulla R, Chan ES, Ravindra P (2011) Biodiesel production from Jatropha curcas: a critical review. Critical Reviews in Biotechnology 31, 53-64. doi:10.3109/07388551.2010.487185

Banilas G, Karampelias M, Makariti I, Kourti A, Hatzopoulos P (2011) The olive DGAT2 gene is developmentally regulated and shares overlapping but distinct expression patterns with DGAT1. Journal of Experimental Botany 62, 521-532. doi:10.1093/jxb/erq286

Burgal J, Shockey J, Lu CF, Dyer J, Larson T, Graham I, Browse J (2008) Metabolic engineering of hydroxy fatty acid production in plants: RcDGAT2 drives dramatic increases in ricinoleate levels in seed oil. Plant Biotechnology Journal 6, 819-831. doi:10.1111/j.1467-7652. 2008.00361.x

Giannoulia K, Haralampidis K, Poghosyan Z, Murphy DJ, Hatzopoulos P (2000) Differential expression of diacylglycerol acyltransferase (DGAT) genes in olive tissues. Biochemical Society Transactions 28, 695-697. doi:10.1042/BST0280695

He XH, Turner C, Chen GQ, Lin JT, McKeon TA (2004) Cloning and characterization of a cDNA encoding diacylglycerol acyltransferase from castor bean. Lipids 39, 311-318. doi:10.1007/s11745-004-1234-2

Hernández ML, Whitehead L, He Z, Gazda V, Gilday A, Kozhevnikova E, Vaistij FE, Larson TR, Graham IA (2012) A cytosolic acyltransferase contributes to triacylglycerol synthesis in sucrose-rescued Arabidopsis seed oil catabolism mutants. Plant Physiology 160, 215-225. doi:10.1104/pp.112.201541

Horsch RB, Fry JE, Hoffmann NL, Eichholtz D, Rogers SG, Fraley RT (1985) A simple and general method for transferring genes into plants. Science 227, 1229-1231. doi:10.1126/science.227.4691.1229

Izard J, Limberger RJ (2003) Rapid screening method for quantitation of bacterial cell lipids from whole cells. Journal of Microbiological Methods 55, 411-418. doi:10.1016/S0167-7012(03)00193-3

Kroon JTM, Wei WX, Simon WJ, Slabas AR (2006) Identification and functional expression of a type 2 acyl-CoA: diacylglycerol acyltransferase (DGAT2) in developing castor bean seeds which has high homology to the major triglyceride biosynthetic enzyme of fungi and animals. Phytochemistry 67, 2541-2549. doi:10.1016/j.phytochem. 2006.09.020

Lardizabal KD, Mai JT, Wagner NW, Wyrick A, Voelker T, Hawkins DJ (2001) DGAT2 is a new diacylglycerol acyltransferase gene family purification, cloning, and expression in insect cells of two polypeptides from Mortierella ramanniana with diacylglycerol acyltransferase activity. Journal of Biological Chemistry 276, 38862-38869. doi:10.1074/jbc.M106168200

Li RZ, Yu KS, Hatanaka T, Hildebrand DF (2010a) Vernonia DGATs increase accumulation of epoxy fatty acids in oil. Plant Biotechnology Journal 8, 184-195. doi:10.1111/j.1467-7652.2009.00476.x

Li RZ, Yu KS, Hildebrand DF (2010b) DGAT1, DGAT2 and PDAT expression in seeds and other tissues of epoxy and hydroxyl fatty acid accumulating plants. Lipids 45, 145-157. doi:10.1007/s11745-010-3385-4

Liu Q, Siloto RM, Snyder CL, Weselake RJ (2011) Functional and topological analysis of yeast acyl-CoA: diacylglycerol acyltransferase 2, an endoplasmic reticulum enzyme essential for triacylglycerol biosynthesis. Journal of Biological Chemistry 286, 13 115-13 126. doi:10.1074/jbc.M110.204412

Lung SC, Weselake R (2006) Diacylglycerolacyltransferase: a key mediator of plant triacylglycerol synthesis. Lipids 41, 1073-1088. doi:10.1007/ s11745-006-5057-y

Maisonneuve S, Bessoule JJ, Lessire R, Delseny M, Roscoe TJ (2010) Expression of rapeseed microsomal lysophosphatidic acid acyltransferase isozymes enhances seed oil content in Arabidopsis. Plant Physiology 152, 670-684. doi:10.1104/pp.109.148247

Mumberg D, Muller R, Funk M (1995) Yeast vectors for the controlled expression of heterologous proteins in different genetic backgrounds. Gene 156, 119-122. doi:10.1016/0378-1119(95)00037-7 
Nykiforuk CL, Furukawa-Stoffer TL, Huff PW, Sarna M, Laroche A, Moloney MM, Weselake RJ (2002) Characterization of cDNAs encoding diacylglycerol acyltransferase from cultures of Brassica napus and sucrose-mediated induction of enzyme biosynthesis. Biochimica et Biophysica Acta 1580, 95-109. doi:10.1016/S1388-1981 (01)00200-1

Ohlrogge J, Browse J (1995) Lipid biosynthesis. The Plant Cell 7, 957-970.

Saha S, Enugutti B, Rajakumari S, Rajasekharan R (2006) Cytosolic triacylglycerol biosynthetic pathway in oilseeds, molecular cloning and expression of peanut cytosolic diacylglycerol acyltransferase. Plant Physiology 141, 1533-1543. doi:10.1104/pp.106.082198

Sandager L, Gustavsson MH, Stahl U, Dahlqvist A, Wiberg E, Banas A, Lenman M, Ronne H, Stymne S (2002) Storage lipid synthesis is nonessential in yeast. Journal of Biological Chemistry 277, 6478-6482. doi:10.1074/jbc.M109109200

Shockey JM, Gidda SK, Chapital DC, Kuan JC, Dhanoa PK, Bland JM, Rothstein SJ, Mullen RT, Dyer JM (2006) Tung tree DGAT1 and DGAT2 have nonredundant functions in triacylglycerol biosynthesis and are localized to different subdomains of the endoplasmicreticulum. The Plant Cell 18, 2294-2313. doi:10.1105/tpc. 106.043695

Siloto RMP, Truksa M, He XH, McKeon T, Weselake RJ (2009) Simple methods to detect triacylglycerol biosynthesis in a yeast-based recombinant system. Lipids 44, 963-973. doi:10.1007/s11745-009$3336-0$

Sorger D, Athenstaedt K, Hrastnik C, Daum G (2004) A yeast strain lacking lipid particles bears a defect in ergosterol formation. Journal of Biological Chemistry 279, 31 190-31 196. doi:10.1074/jbc.M403251200

Stone SJ, Levin MC, Farese RV (2006) Membrane topology and identification of key functional amino acid residues of murine acyl-CoA: diacylglycerol acyltransferase-2. Journal of Biological Chemistry 281, 40 273-40 282. doi:10.1074/jbc.M607986200

Tamura K, Peterson D, Peterson N, Stecher G, Nei M, Kumar S (2011) MEGA5: molecular evolutionary genetics analysis using maximum likelihood, evolutionary distance, and maximum parsimony methods. Molecular Biology and Evolution 28, 2731-2739. doi:10.1093/molbev/ msr121

Turchetto-Zolet AC, Maraschin FS, de Morais GL, Cagliari A, Andrade CM, Margis-Pinheiro M, Margis R (2011) Evolutionary view of acyl-CoA diacylglycerol acyltransferase (DGAT), a key enzyme in neutral lipid biosynthesis. BMC Evolutionary Biology 11, 263-276. doi:10.1186/ 1471-2148-11-263
Wagner M, Hoppe K, Czabany T, Heilmann M, Daum G, Feussner I, Fulda M (2010) Identification and characterization of an acyl-CoA:diacylglycerol acyltransferase 2 (DGAT2) gene from the microalga $O$. tauri. Plant Physiology and Biochemistry 48, 407-416. doi:10.1016/j.plaphy.2010. 03.008

Wang J, Li R, Lu D, Ma S, Yan Y, Li W (2009) A quick isolation method for mutants with high lipid yield in oleaginous yeast. World Journal of Microbiology \& Biotechnology 25, 921-925. doi:10.1007/s11274-0099960-2

Worrall D (1998) PCR analysis of transgenic tobacco plants. Methods in Molecular Biology 81, 417-423.

Xu J, Francis T, Mietkiewska E, Giblin EM, Barton DL, Zhang Y, Zhang M, Taylor DC (2008) Cloning and characterization of an acyl-CoAdependent diacylglycerol acyltransferase $1(D G A T l)$ gene from Tropaeolum majus, and a study of the functional motifs of the DGAT protein using site-directed mutagenesis to modify enzyme activity and oil content. Plant Biotechnology Journal 6, 799-818. doi:10.1111/j.14677652.2008.00358.x

Xu RH, Wang RL, Liu AZ (2011) Expression profiles of genes involved in fatty acid and triacylglycerol synthesis in developing seeds of Jatropha (Jatropha curcas L.). Biomass and Bioenergy 35, 1683-1692. doi:10.1016/j.biombioe.2011.01.001

Yu K, Li R, Hatanaka T, Hildebrand D (2008) Cloning and functional analysis of two type 1diacylglycerol acyltransferases from Vernonia galamensis. Phytochemistry 69, 1119-1127. doi:10.1016/j.phytochem. 2007.11.015

Zhang M, Fan J, Taylor DC, Ohlrogge JB (2009) DGAT1 and PDAT1 acyltransferase shave overlapping functions in Arabidopsis triacylglycerol biosynthesis and are essential for normal pollen and seed development. The Plant Cell 21, 3885-3901. doi:10.1105/ tpc. 109.071795

Zheng P, Allen WB, Roesler K, Williams ME, Zhang S, Li J, Glassman K, Ranch J, Nubel D, Solawetz W, Bhattramakki D, Llaca V, Deschamps S, Zhong GY, Tarczynski MC, Shen B (2008) A phenylalanine in DGAT is a key determinant of oil content and composition in maize. Nature Genetics 40, 367-372. doi:10.1038/ng.85

Zou JT, Wei YD, Jako C, Kumar A, Selvaraj G, Taylor DC (1999) The Arabidopsis thaliana TAG1 mutant has a mutation in a diacylglycerol acyltransferase gene. The Plant Journal 19, 645-653. doi:10.1046/j.1365313x.1999.00555.x 